\title{
A REVIEW ON VITEX NEGUNDO LINN
}

\author{
Ishwarya.R, Kousalya.N, Logeshwaran.V, Sabarinath.K, Sandhiya.S ,Arun.P \\ Department of Biotechnology, \\ Dr. N. G. P Arts and Science College, Coimbatore-48
}

\begin{abstract}
Vitex negundo belongs to the family verbenaceae, is a aromatic shrub. This plant is commonly used for traditional medicines to treat various diseases. All parts include leaves, root, bark, seeds and flowers are used to treat diseases. It contains number of phytochemicals as secondary metabolites compare to other parts leaves contain number of phytochemicals these play a vital role in tradicinal medicines. Plant extracts of Vitex negundo possess different activities like anti-microbial, anti inflammatory, anti- oxidant, anti- pyretic and others. It is also used as a larvidal, mosquito repellent and insecticidal. This review aims to presenting the role of phytochemicals and its therapeutic uses to develop the modern medicine.
\end{abstract}

Keywords-Vitex negundo,Verbenaceae,Traditional medicine, Phytochemicals

\section{INTRODUCTION}

Vitex negundo linn is a aromatic shrub, is commonly called as nirgundi or nochi is found throughout the India is commonly grows in waste lands [1]. Its leaves, barks, roots, fruits and flowers are used as medicinal purposes in all over the India [2]. Phytochemical analysis of Vitex negungo confirms the presence of Trepenoids, Flavonoids, Steroids, Anthroquione,Glycosides,

Sugars, Alkaloid, Quinones, Phenols,Tannins, Saponins, Coumarin. [3]. It has a various medicinal properties particularly in the treatment of anti-arthritic, fungal diseases, antioxidant and other activities [4].

\section{PLANT DESCRIPTION}

Vitex negundo is a woody, and large shrubs or small trees up to $2.5-4.5 \mathrm{~m}$ high, with quadrangular branches. The plant has pungent, bitter, acrid taste. Leaves are 3-5 penta foliate and the leaflets are arranged palmately and terminal leafletsare long $(5-10 \mathrm{~cm})$ acute with petiolate $(1-1.3 \mathrm{long})$, hairy beneath and both the ends are pointed. Flowers are in pedunculate branched tomentose cymes, which are bluish purple in colour and the fruits areround, succulent and black on ripening with four seeds. [5]

a) Geographical indication:

Vitex negundo is distributed throughout the India;altitude of $1500 \mathrm{~m}$ in the outer Himalayas It is grown commercially as a crop in parts of Asia, Europe, North America and West Indies. [5] b) Classification:

Kingdom - Plantae

Subkingdom - Tracheobionta

Super division - Spermatophyte

Division - Magnoliophyta

Class - Magnoliopsida

Subclass - Asteridae

Order - Lamiales

Family - Verbenaceae

Genus - Vitex

Species - Negundo

\section{ACTIVITIES}

a) Antimicrobial activity:

Microbes are present everywhere which causes infectious diseases to humans in world wide. Vitex negundo Linn acts on both gram positive and gram negative organism. The plant extract acts as a antimicrobial agent. [6]

b) Anti-inflammatory activity:

Anti inflammatory property is to treat inflammation. The anti-inflammatory property of Vitex negundo is confirmed by clinical trials on rat. The leaf extract of Vitex negundo prevents carrageenan-induced rat paw edema and formaldehyde-induced rat paw edema. [1]

c) Antipyretic activity:

Pyrexia or fever is caused by secondary impact of infection, graft rejection and other diseased states. Antipyretic are used to reduce the body temperature. The presence of flavonoids in the Vitex negundo is responsible for the antipyretic activity. The antipyretic property of Vitex negundo shows the good result in albino rats induced yeast hyper pyrexia and it was compared to standard drug paracetamol. [7]

d) Anti micro filarial activity:

In India around 48 million people are affected by filariasis. It is a vector - borne parasitic disease a adult worm lives in the infected individual for several years. Vitex negundo plant extract as antifilarial activity against Setariacervi. [8]

e) Anti -oxidant activity:

Free radicals causes cell damage due to the singlet oxygen or hydrogen peroxide leads to oxidative stress. This stress plays an important role in the pathogenesis of certain cancers and atherosclerosis. Fruits, vegetables and herbs are rich sources of antioxidants such as phenolic compounds, flavonoids, carotenoids, tocopherol and ascorbic acid. 


\section{International Journal of Engineering Applied Sciences and Technology, 2019 \\ Vol. 4, Issue 8, ISSN No. 2455-2143, Pages 195-198 \\ Published Online December 2019 in IJEAST (http://www.ijeast.com)}

Some of the antioxidant present in vitex negundo are Catechin,Epicatechin Quercetin ,Myricetin Kaempferol ,Naringenin , $\beta$-carotene ,Tocopherol [9]

\begin{tabular}{|c|c|c|c|}
\hline S.NO & ACTIVITY & $\begin{array}{c}\text { ACTION } \\
\text { AGAINST }\end{array}$ & REFERNCES \\
\hline 1 & $\begin{array}{c}\text { Anti } \\
\text { bacterial }\end{array}$ & \begin{tabular}{l}
\multicolumn{1}{c}{$\begin{array}{c}\text { Gram negative } \\
\text { bacteria: }\end{array}$} \\
Escherichia coli, \\
Pseudomonas \\
aeruginosa, Vibrio \\
cholera, \\
Salmonella typhi, \\
Shigella boydii, \\
Shigella \\
dysenteriae, ,Vibrio \\
mimicus \\
,Salmonella \\
paratyphi,Vibrio \\
parahemolyticus \\
Gram positive \\
bacteria \\
Bacillus subtilis, \\
Streptococcus \\
pyogenes \\
Staphylococcus \\
aureus,Bacillus \\
cereus,Bacillus \\
megaterium \\
,Sarcina lutea
\end{tabular} & {$[12,13]$} \\
\hline 2 & Anti pyretic & $\begin{array}{l}\text { Yeast induced } \\
\text { hyper pyrexia }\end{array}$ & [7] \\
\hline 3 & Anti filarial & $\begin{array}{c}\text { Setaria cervi } \\
\text { (microfilarial } \\
\text { parasite) } \\
\text { Brugia malayi }\end{array}$ & {$[8,11]$} \\
\hline 4 & $\begin{array}{l}\text { Larvicidal } \\
\text { and } \\
\text { mosquito } \\
\text { repellent }\end{array}$ & $\begin{array}{l}\text { Culex } \\
\text { quinquefasciatus, } \\
\text { Anopheles } \\
\text { stephensi, Aedes } \\
\text { aegypti, Culex } \\
\text { tritaeniorhynchus } \\
\text {,Andrographis } \\
\text { paniculata }\end{array}$ & {$[14,15]$} \\
\hline 5 & Anti fungal & $\begin{array}{l}\text { C.albicans, } \\
\text { C.krusei, } \\
\text { C.parapsilosis, } \\
\text { C. tropicalis } \\
\text { C.glabrata, } \\
\text { Aspergillus flavus, } \\
\text { Microsporum canis } \\
\text { and } \\
\text { Fusarium solani }\end{array}$ & {$[16,17]$} \\
\hline 6 & $\begin{array}{r}\text { Anti } \\
\text { inflammator } \\
\text { Acti }\end{array}$ & $\begin{array}{c}\text { Carrageenin } \\
\text { induced hind paw } \\
\text { ies of Vitex negundo }\end{array}$ & [1] \\
\hline
\end{tabular}

\section{f) Hepatoprotective Activity:}

Vitex negundo contains negundoside and agundoside compounds are used to treat hepatic diseases.[4]

g) Anticonvulsant activity

The petroleum ether and butanol leaf extract have shown protection against electro shock seizures, whereas root extract has shown little effect. Petroleum ether extract of root could only provide protection against leptazole induced convultions, whereas methanolic leaf extract showed significant protection against both strychnine and leptazole induced convultions . [10]

\section{PHYTOCHEMICALS}

a) Leaves:

Aromadendrene oxide-(1) ,n-Hexadecaonoic acid 4H-Pyran-4-one, 2,3-dihydro-3,5-dihydroxy-6-methyl- 2,4Pentadien-1-ol, 3-propyl-, (2Z)- ,D-Glucose, 6-O-_-Dgalactopyranosyl- ,Ascaridole epoxide ,d-Mannose , Butane, 1,1-diethoxy-3-methyl, Hexanoic acid, ethyl ester Propane, 1,1,3-triethoxy- ,2,3-Dihydrothiophene 1,1-dioxide ,Ledol , Ethyl iso-allocholate (7_-Isopropenyl-4,5dimethyloctahydroinden-4-yl)methanol, ,Azulene, 1,4dimethyl-7-(1-methylethyl)- , Ethanol, 2-(9-octadecenyloxy)-, (Z)- , 4,9-Decadienoic acid, 2-nitro-, ethyl ester ,Hexadecanoic acid, ethyl ester 10, 13-Octadecadiynoic acid, methyl ester ,4-Decynoic acid, methyl ester Caryophyllene ,Benzoic acid, 3-hydroxy- , 2-Methyl-4-(2,6,6trimethylcyclohex-1-enyl)but-2-en-1-ol 6,9,12,15Docosatetraenoic acid, methyl ester ,Phytol ,Ethanol, 2-(9,12octadecadienyloxy)-, (Z,Z)- ,9,12,15-Octadecatrienoic acid, (Z,Z,Z)-,12-Bromo-13-hydroxy-2,5,9,13-tetramethyltetradeca4,8-dienoic acid, methyl ester Vitamin E, negundoside, agnuside, vitegnoside [18]

1H-indene, cyclododecanol, patchoulane, 1,2dihexylcyclopropene-3-carboxylic acid, 2-heptenoic acid, (+) - aromadendrene, trans-caryophyllene, 7-oxabicyclo heptane, cyclohexane, farnesol, pentadecane and 1-octanol. Tetramethoxyflavone, trimethoxyflavone, ascerosin and 5glucosylrhamnoside; casticin, chrysoplenol D, lutcolin,isooxientin, sabinene,p-cymene, M-phelladune, Nterpinene, global andviridiflorol4; mono and sesquiterpenes5; viridiflorol, M-eudesmol and M-caryophyllene6.[19]

Pentacyclic triterpenoids, betulinic acid (3_hydroxylup-20-(29)-en-28-oic acid), and ursolic acid(2_hydroxyurs-12-en-28-oic acid) .[20]

b) Seeds:

Ono3 $\beta$-Acetoxyolean-12-en-27-oic acid; $2 \alpha, \quad 3 \alpha-$ dihydroxyoleana-5,12-dien-28-oic acid; $2 \beta, 3 \alpha$ diacetoxyoleana-5,12-dien-28-oic acid; $2 \alpha, 3 \beta$-diacetoxy-18hydroxyoleana-5,12-dien-28-oic acid 6-Hydroxy-4-(4hydroxy-3- methoxy-phenyl)-3-hydroxymethyl-7-methoxy3,4- dihydro-2-naphthaldehyde $\beta$-Sitosterol, $p$-hydroxybenzoic acid, $n$-tritriacontane, nhentriacontane, $n$-pentatriacontane, and n-nonacosane. [21] 


\section{International Journal of Engineering Applied Sciences and Technology, 2019 \\ Vol. 4, Issue 8, ISSN No. 2455-2143, Pages 195-198 \\ Published Online December 2019 in IJEAST (http://www.ijeast.com)}

Phenyldihydronaphthalene-type lignan, vitedoin A, a new phenylnaphthalene-type lignan alkaloid, vitedoamine A , and a new trinorlabdane-type diterpene, vitedoin B.[22]

\section{c) Essential oil:}

Ethyl-hexadecenoate; $\alpha$-selinene, germacren-4ol; caryophyllene epoxide, (E)-nerolidol, $\beta$-selinene, $\alpha$ cedrene, germacrene $\mathrm{D}$, hexadecanoic acid, p-cymene and valencene, viridiflorol, $\beta$-caryophyllene, sabinene , 4terpineol , $\gamma$-terpinene , caryophyllene oxide , 1-oceten-3-ol , and globulol. [23]

\section{CONCLUSION}

\begin{tabular}{|c|c|c|}
\hline S.NO & COMPOUND & ACTIVITY \\
\hline 1 & $\begin{array}{l}\text { 4H-Pyran-4-one, 2,3- } \\
\text { dihydro- } \\
\text { 3,5-dihydroxy-6-methyl- }\end{array}$ & $\begin{array}{l}\text { Antimicrobial, } \\
\text { inflammatory }\end{array}$ \\
\hline 2 & $\begin{array}{l}\text { D-Glucose,6-O-_- } \\
\text { Dgalactopyranosyl- }\end{array}$ & Preservative \\
\hline 3 & $\begin{array}{l}\text { Hexadecanoic acid, } \\
\text { ethyl ester }\end{array}$ & $\begin{array}{l}\text { Antioxidant, } \\
\text { hypocholesterolemic } \\
\text { nematicide, pesticide, anti } \\
\text { androgenic flavor, } \\
\text { hemolytic, 5-Alpha } \\
\text { reductase inhibitor }\end{array}$ \\
\hline 4 & Caryophyllene & $\begin{array}{l}\text { Anti-tumor, analgesic, } \\
\text { antibacterial, } \\
\text { antiinflammatory, } \\
\text { sedative, fungicide }\end{array}$ \\
\hline 5 & Benzoic acid, 3-hydroxy & Antimicrobial \\
\hline 6 & Ledol & $\begin{array}{l}\text { Antimicrobial, anti- } \\
\text { inflammatory }\end{array}$ \\
\hline 7 & $\begin{array}{l}\text { Aromadendrene oxide- } \\
\text { (1) }\end{array}$ & $\begin{array}{l}\text { Anti-tumor, analgesic, } \\
\text { antibacterial, } \\
\text { antiinflammatory, sedative }\end{array}$ \\
\hline 8 & n-Hexadecaonoic acid & $\begin{array}{l}\text { Antioxidant, } \\
\text { hypocholesterolemic } \\
\text { nematicide, pesticide, anti } \\
\text { androgenic flavor, } \\
\text { hemolytic, 5-Alpha } \\
\text { reductase inhibitor }\end{array}$ \\
\hline 9 & Phytol & $\begin{array}{l}\text { Antimicrobial, anticancer, } \\
\text { antiinflammatory, diuretic }\end{array}$ \\
\hline 10 & Activity of Phytoc & $\begin{array}{l}\text { Antiageing, analgesic, } \\
\text { antidiabaticantiinflammato } \\
\text { ry, antioxidant, } \\
\text { antidermatitic, } \\
\text { antileukemic, antitumor, } \\
\text { anticancer, } \\
\text { hepatoprotective, } \\
\text { vnnchnlesternlemic, } \\
\text { emicals[24] }\end{array}$ \\
\hline & & $\begin{array}{l}\text { munusuvine, } \\
\text { anticoronary }\end{array}$ \\
\hline
\end{tabular}

Vitex negundo is a natural gift from god it possess wide application in traditional medicines. All parts of the plants include leaf, roots, flowers, seeds and barks are used to treat many infectious diseases. It contains multiple numbers of phytochemicals as secondary metabolites, each plays the unique role to treat diseases. Caryophyllene, ledol, nishindaside, mussaenosidic acids, vitedoin, negundin and vitexin are some of the important phytochemicals in the plants.

This plant is known to possess ant-inflammatory, anti-microbial, anti pyretic, anticonvulsunt, insecticidal and it is highly hepatoprotective. Besides, this medicinal properties it is also act as mosquito repellent and larvicidal activity.

\section{ACKNOWLEDGEMENT}

The author express their gratitude towards the host institution Dr.N.G.P. Arts and Science College, Management, Principal, Deans, Head of the Department, Guide and all other staffs of Department of Biotechnology for rendering all the facilities for support. Communication no: Dr NGPASC 2019. 2020 BS017.

\section{REFERENCES}

1. Tandon, V. R., \& Gupta, R. K. (2006). Antiinflammatory activity and mechanism of action of Vitex negundo Linn. International journal of pharmacology, 2(3), 303-8.

2. Mohanraj, S., Vanathi, P., Sowbarniga, N., \& Saravanan, D. (2012). Antimicrobial effectiveness of Vitex negundo leaf extracts.

3. Anbuselvan. S and Muralikrishnan. V, (2013). Phytochemical evaluation and Antimicrobial activity of Vitex negundo(1.) leaves extracts. International journal of research in Pharmaceutical and Nano sciences. , 44 - 56.

4. Kambham Venkateswarlu, Vitex negundo: Medicinal Values, Biological Activities, Toxicity Studies and Phytopharmacological Actions (2012). International Journal of Pharmaceutical and Phytopharmacological Research, 2(2): 126-133

5. Suganthi, N., \& Dubey, S. (2016). Phytochemical constituents and pharmacological activities of Vitex negundo Linn. Journal of Chemical and Pharmaceutical Research, 8(2), 800-807.

6. Meena ShamraoDeogade, Tarulata Pandya1, KethamakkaShivarama Prasad Kunal Kale, NilimaTankhiwale, (2016). Antimicrobial activity of Vitex negundolinn (nirgundi) leaves extract, original article - pharmacological research, vol 2(4).

7. Miskin, N., Manjunath, K. P., Bhandarkar, A., Bolakatti, G., \& Katagi, M. S. (2012). Antipyretic activity of Vitex negundo Linn Leaves extracts. RGUHS J Pharm Sci, 2(2), 78-81. 


\section{International Journal of Engineering Applied Sciences and Technology, 2019 Vol. 4, Issue 8, ISSN No. 2455-2143, Pages 195-198 \\ Published Online December 2019 in IJEAST (http://www.ijeast.com)}

8. Sahare, K. N., \& Singh, V. (2013). Antifilarial activity of ethyl acetate extract of Vitex negundo leaves in vitro. Asian Pacific journal of tropical medicine, 6(9), 689-692.

9. Zargar, M., Azizah, A. H., Roheeyati, A. M., Fatimah, A. B., Jahanshiri, F., \& Pak-Dek, M. S. (2011). Bioactive compounds and antioxidant activity of different extracts from Vitex negundo leaf. Journal of Medicinal Plants Research,5(12), 2525-2532.

10. Basri, F., Sharma, H. P., Firdaus, S., Jain, P., \& Ranjan, A. (2014). A review of ethnomedicinal plantVitex negundo Linn. International Journal of Advanced Research, 2(3), 882-894

11. Zheng, C. J., Li, H. Q., Ren, S. C., Xu, C. L., Rahman, K., Qin, L. P., \& Sun, Y. H. (2015). Phytochemical and pharmacological profile of Vitex negundo. Phytotherapy research, 29(5), 633-647.

12. Prashith Kekuda TR, Manasa M , Poornima G , Abhipsa V , Rekha C ,Shivaleela P. Upashe2 and Raghavendra HL (2013), Antibacterial, Cytotoxic and Antioxidant Potential of Vitex Negundo Var.Negundo and Vitex Negundo Var. Purpurascens - A Comparative Study, Science, Technology and Arts Research Journal 2(3): 59-68

13. Chowdhury, J. A., Islam, M. S., Asifuzzaman, S. K., \& Islam, M. K. (2009). Antibacterial and cytotoxic activity screening of leaf extracts of Vitex negundo (Fam: Verbenaceae). Journal of pharmaceutical sciences and research, 1(4), 103.

14. Vasanth Raj, P., Raghu Chandrasekhar, H., Dhanaraj, S. A., Vijayan, P., Nitesh, K., Subrahmanyam, V. M., ... \& Raj, M. P. V. MOSQUITO LARVICIDAL ACTIVITY OF VITEX NEGUNDO.

15. Karunamoorthi, K., Ramanujam, S., \& Rathinasamy, R. (2008). Evaluation of leaf extracts of Vitex negundo L.(Family: Verbenaceae) against larvae of Culex tritaeniorhynchus and repellent activity on adult vector mosquitoes. Parasitology Research, 103(3), 545.

16. Panda, S. K., Behera, B., \& Dutta, S. K. (2009). Anticandidal activity of Vitex negundo L.: An ethnomedicinal plant. Journal of Pure and Applied Microbiology, 3(2), 777-784.

17. Mahmud, S., Shareef, H., Farrukh, U., Kamil, A., \& Rizwani, G. H. (2009). Antifungal activities of Vitex negundo Linn. Pak. J. Bot, 41(4), 1941-1943.

18. Gautam, L. M., Shrestha, S. L., Wagle, P., \& Tamrakar, B. M. (2008). Chemical constituents from Vitex negundo (Linn.) of nepalese origin. Scientific world, 6(6), 27-32.

19. Sahayaraj, K., \& Ravi, C. (2008). Preliminary phytochemistry of Ipomea carnea jacq. and Vitex negundo Linn. leaves. Int. J. Chem. Sci, 6(1), 1-6.
20. Chandramu, C., Manohar, R. D., Krupadanam, D. G., \& Dashavantha, R. V. (2003). Isolation, characterization and biological activity of betulinic acid and ursolic acid from Vitex negundo L. Phytotherapy Research: An International Journal Devoted to Pharmacological and Toxicological Evaluation of Natural Product Derivatives, 17(2), 129-134.

21. Gill, B. S., Mehra, R., \& Kumar, S. (2018). Vitex negundo and its medicinal value. Molecular biology reports, 45(6), 2925-2934.

22. Ono, M., Nishida, Y., Masuoka, C., Li, J. C., Okawa, M., Ikeda, T., \& Nohara, T. (2004). Lignan derivatives and a norditerpene from the seeds of Vitex negundo. Journal of natural products, 67(12), 2073-2075.

23. Balasubramani, S., Rajendhiran, T., Moola, A. K., \& Diana, R. K. B. (2017). Development of nanoemulsion from Vitex negundo L. essential oil and their efficacy of antioxidant, antimicrobial and larvicidal activities (Aedes aegypti L.). Environmental Science and Pollution Research, 24(17), 15125-15133.

24. Kumar, P. P., Kumaravel, S., \& Lalitha, C. (2010). Screening of antioxidant activity, total phenolics and GC-MS study of Vitex negundo. African Journal of Biochemistry Research, 4(7), 191-195. 